\title{
Aterosclerose Precoce na População Pediátrica Infetada pelo VIH: Revisão da Literatura e Abordagem Clínica
}

\author{
Premature Atherosclerosis in HIV-Infected Pediatric \\ Patients: Literature Review and Clinical Approach
}

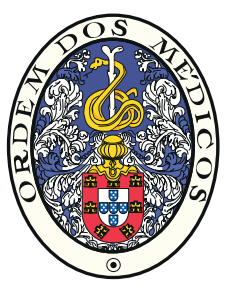

Paula MARTINS $\triangle^{1,2}$, Eduardo CASTELA ${ }^{1}$, Graça ROCHA ${ }^{3}$, Cristina SENA ${ }^{4}$, Raquel SEIÇA 4

Acta Med Port 2017 Oct;30(10):742-749 - https://doi.org/10.20344/amp.8726

\section{RESUMO}

As crianças e adolescentes infetados pelo vírus da imunodeficiência humana representam um grupo pediátrico com risco acrescido de doença cardiovascular precoce. O próprio vírus, a terapêutica antirretrovírica e os estilos de vida estabelecem um complexo conjunto interatuante de fatores que promove a aterosclerose acelerada. Este processo é provavelmente mediado por dislipidémia, alterações do metabolismo glicídico, inflamação crónica com disfunção endotelial e um estado pro-trombótico. A abordagem clínica desta população para prevenção cardiovascular é baseada sobretudo num tratamento eficaz da infeção, na redução dos fatores de risco modificáveis e na promoção de hábitos saudáveis.

Palavras-chave: Antirretrovirais; Aterosclerose; Criança; Factores de Risco; Infecções por HIV

\section{ABSTRACT}

Human immunodeficiency virus infected children and adolescents are a pediatric group with increased risk of premature cardiovascular disease. The virus itself, the antiretroviral therapy and the lifestyle establish a complex interplay of factors that promotes an accelerated atherosclerosis. This process is probably mediated by dyslipidaemia, dysregulation of glucose metabolism, lipodystrophy, inflammation, endothelial dysfunction and a prothrombotic state. The clinical approach to this population in terms of cardiovascular prevention is mainly based on efficient treatment of the infection, reduction of the modifiable risk factors and promotion of lifestyle changes.

Keywords: Anti-Retroviral Agents; Atherosclerosis; Child; HIV Infections; Risk Factors

\section{INTRODUÇÃO}

O processo aterosclerótico inicia-se no feto e progride lentamente ao longo da vida, sendo responsável por cerca de $50 \%$ da mortalidade nos países europeus. ${ }^{1}$

$\mathrm{Na}$ população infetada com vírus da imunodeficiência humana ( $\mathrm{VIH})$, a aterosclerose ocorre de uma forma acelerada, podendo aumentar a morbilidade e diminuir a sobrevida deste grupo. De facto, o risco de enfarte agudo do miocárdio é $50 \%$ superior nos seropositivos, quando comparados com a população em geral, mesmo após efectuado o ajuste para os outros fatores de risco cardiovasculares contemplados no score de Framingham. ${ }^{2} \mathrm{~A}$ incidência de acidente vascular cerebral também é mais elevada nos doentes infetados, como comprovado por Marcus et al (2014), sobretudo se tiverem uma elevada carga vírica e/ ou uma baixa contagem de células CD4, ${ }^{3}$ correspondendo a uma fase avançada da doença.

Nas crianças e adolescentes infetados, o leito vascular arterial exibe precocemente alterações estruturais e funcionais próprias de aterosclerose sub-clínica.4-10 Múltiplos fatores parecem convergir para propiciar um meio pró-aterosclerótico no contexto da infeção VIH. Reconhecer esta realidade é indispensável para um correcto acompanhamento destes doentes em idade pediátrica, evitando complicações cardiovasculares futuras.

\section{Estado pró-aterosclerótico}

Muita da informação existente sobre o processo aterosclerótico no contexto da infeção VIH provém de estudos efectuados em adultos. No entanto, os dados científicos disponíveis relativos à população pediátrica, aponta para mecanismos pró-ateroscleróticos idênticos, ainda que as manifestações clínicas escasseiem nesta faixa etária.

Na população infetada parece haver uma maior prevalência dos fatores de risco cardiovascular 'clássicos' como o tabagismo, ${ }^{11,12}$ a hipertensão arterial, a dislipidémia, a insulinorresistência e/ou diabetes. Este predomínio de fatores de risco é só parcialmente justificado pelos estilos de vida e pela genética, desempenhando provavelmente a infeção crónica e a terapêutica antirretroviral uma influência preponderante (Fig. 1). 6,13,14

Em algumas casuísticas de doentes infetados, a hipertensão arterial tem surgido como mais prevalente do que nos grupos controlo, ${ }^{15-18}$ relacionando-se com o índice de massa corporal, a idade, o sexo, o perfil lipídico, a distribuição da gordura corporal e a insulinorresistência. ${ }^{18-20} \mathrm{~A}$ duração da terapia antirretrovírica combinada e o tipo de esquema terapêutico adotado parecem também influenciar a probabilidade de desenvolver hipertensão. ${ }^{16,19,21}$ Todavia, estes resultados não são universais, existindo séries em que os valores tensionais são idênticos nos dois grupos,

\footnotetext{
1. Serviço de Cardiologia Pediátrica. Centro Hospitalar e Universitário de Coimbra. Coimbra. Portugal.

2. Faculdade de Medicina. Universidade de Coimbra. Coimbra. Portugal.

3. Serviço de Pediatria Ambulatória. Centro Hospitalar e Universitário de Coimbra. Coimbra. Portugal.

4. Laboratório de Fisiologia. Instituto de Imagem Biomédica e Ciências da Vida (IBILI). Faculdade de Medicina. Universidade de Coimbra. Coimbra. Portugal.

$\square$ Autor correspondente: Paula Martins. paula_mrtns@yahoo.com

Recebido: 23 de janeiro de 2017 - Aceite: 25 de setembro de 2017| Copyright @ Ordem dos Médicos 2017
} 


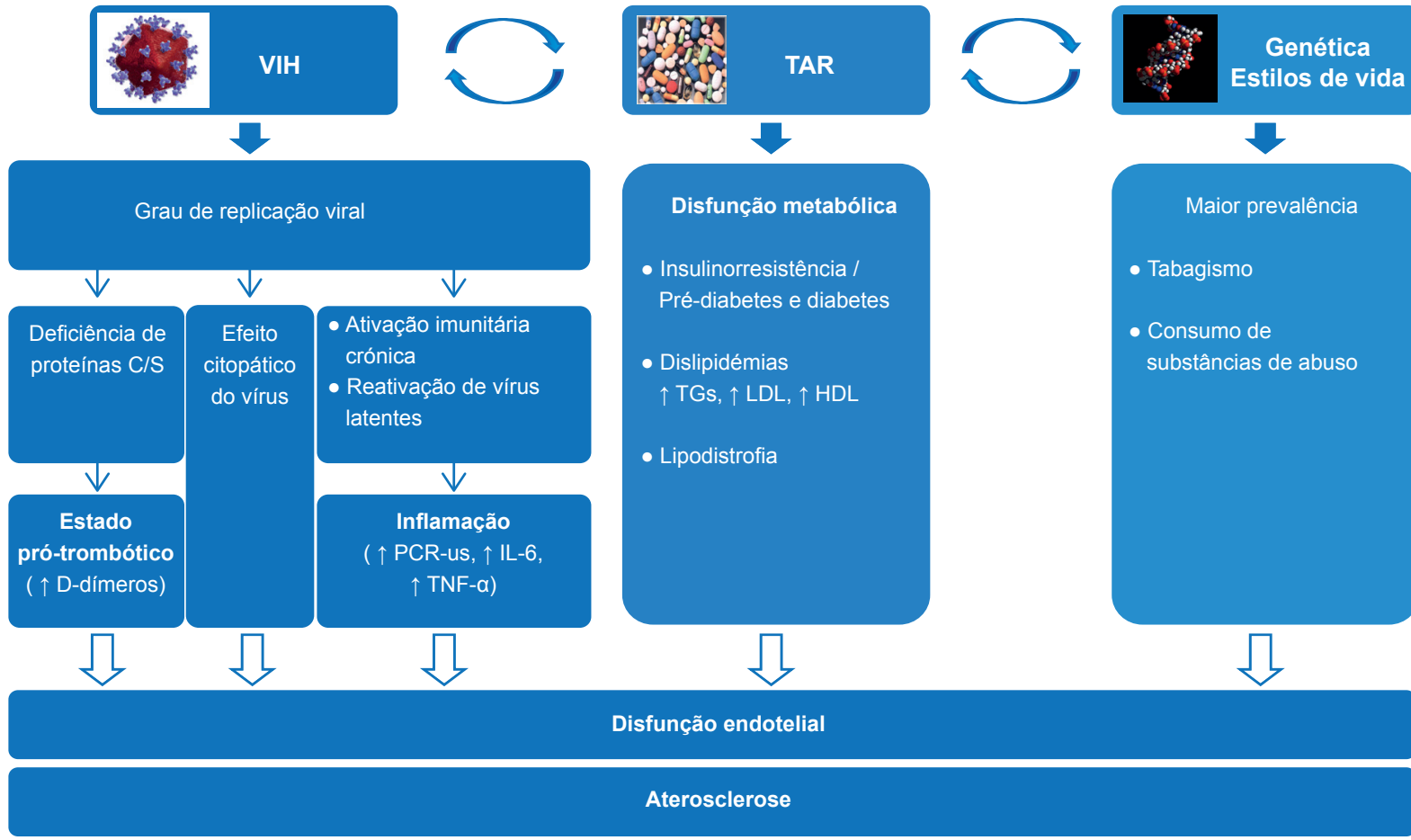

Figura 1 - Esquema simplificado da etiopatogénese da aterosclerose no contexto da infeção pelo vírus da imunodeficiência humana $(\mathrm{VIH})$

As imagens utilizadas foram obtidas a partir da Wikimedia Commons. Imagem do VIH by BruceBlaus (Own work) [CC BY-SA 4.0 (http://creativecommons.org/licenses/by-sa/4.0)], via Wikimedia Commons. Imagem de comprimidos by RayNata - Own work, GFDL, https://commons. wikimedia.org/w/index.php?curid=5280969. Imagem da dupla hélix de ADN by UdeOwn work, https://commons. wikimedia.org/wiki/File:DNA Helix CPK.jpg?uselang=pt.

PCR-us: proteína C reativa ultrassensível; IL-6: interleucina - 6; TNF-a: fator de necrose tumoral-alfa; TGs: triglicerídeos; LDL: lipoproteína de baixa densidade; HDL: lipoproteína de alta densidade; TAR: terapêutica antirretrovírica

ou até inferiores nos indivíduos seropositivos sob terapêutica antirretrovírica. ${ }^{14,22,23}$ A incongruência das conclusões obtidas prende-se, provavelmente, com a heterogeneidade clínica das populações analisadas, com as diferenças no desenho dos estudos e com as metodologias escolhidas. Em idade pediátrica, destaca-se o trabalho de Chatterton-Kirchmeier publicado em 2015 no Pediatric Infectious Disease Journal. Foram estudadas cento e sete crianças e adolescentes infetados, com idades compreendidas entre os dois e 17 anos. A prevalência de hipertensão neste grupo foi de $20 \%$ e foram identificados alguns fatores predisponentes como o tabagismo, o sexo masculino e a presença de certas comorbilidades como a síndrome do ovário poliquístico, a apneia obstrutiva do sono e o consumo de cocaína. Não se identificou contudo correlação entre hipertensão arterial e um número elevado de cópias de ARN viral ou a terapêutica antirretrovírica utilizada. ${ }^{24}$

Mais clara e mais reprodutível nas diferentes séries tem sido a relação entre infeção VIH/terapêutica antirretrovírica e a presença de disfunção metabólica traduzida por lipodistrofia, dislipidémia e/ou insulinorresistência (Fig. 1).

A lipodistrofia refere-se ao processo de redistribuição anómala da gordura corporal, com redução da massa gorda em certas áreas (lipoatrofia), e acumulação indesejada noutras zonas do corpo (lipohipertrofia). Este fenómeno pode ocorrer em cerca de $34 \%$ dos doentes pediátricos, sendo mais comum em adolescentes do que em crianças pré-puberes. ${ }^{25}$ Para além das implicações cardiovascula- res, a lipodistrofia pode ser uma potencial fonte de estigmatização, influenciando negativamente a auto-estima dos jovens afetados. É por isso fator predisponente para depressão e má adesão à terapêutica.

A lipoatrofia acomete sobretudo a gordura subcutânea da face, membros e nádegas, ou seja, o tecido adiposo com uma localização mais periférica. Surge com maior prevalência no sexo masculino, na raça caucasiana e nos indivíduos com doença avançada. Está preferencialmente associada ao uso de inibidores da transcriptase reversa análogos da timidina, como a estavudina e a zidovudina. Estes fármacos provocam depleção do ácido desoxirribonucleico (ADN) mitocondrial, com consequente disfunção da cadeia respiratória, redução da produção energética, diminuição da lipogénese e aumento da apoptose adipocitária. A utilização de esquemas terapêuticos sem os análogos de timidina permite reduzir o risco de lipoatrofia em cerca de um terço. Este risco não é totalmente anulado, uma vez que a própria infeção vírica tem também a capacidade de afetar o ADN mitocondrial. ${ }^{26}$

A lipohipertrofia, cujo processo etiopatogénico ainda não está bem esclarecido, caracteriza-se por uma distribuição central da adiposidade, com acumulação da gordura no abdómen, tórax e região dorsocervical. É frequentemente um efeito secundário dos inibidores da protease, embora possa existir na ausência de terapêutica antirretrovírica. ${ }^{25-34}$ Num subgrupo minoritário de doentes, a lipohipertrofia pode coexistir com a lipoatrofia, existindo um padrão misto 
de apresentação.

A dislipidémia, por sua vez, está presente em $40 \%$ a $75 \%$ das crianças mais velhas e adolescentes com antecedentes de terapêutica antirretrovírica prolongada. A sua prevalência varia com o esquema antirretrovírico escolhido, com a duração do tratamento e com os estilos de vida da amostra estudada. O perfil lipídico tem tipicamente um aumento do colesterol low density lipoproteins (LDL), redução do colesterol high density lipoproteins (HDL) e hipertrigliceridémia. Trata-se de um perfil reconhecidamente aterogénico e que está relacionado sobretudo com o uso de inibidores da protease..$^{35-38}$ Investigação laboratorial demonstrou que o efeito hiperlipidemiante destes fármacos é provavelmente devido à inibição da atividade de certas proteínas-chave do metabolismo lipídico, como por exemplo o recetor de LDL (LDLR) e a proteína relacionada com LDLR (LRP). Essa inibição diminuiria a capacidade dos hepatócitos e das células endoteliais de removerem da circulação os quilomicrons (ricos em triglicerídeos) e as partículas LDL, aumentando assim o seu teor plasmático. ${ }^{28,39}$ Alguns inibidores da transcriptase reversa, sobretudo a estavudina e o efavirenz, têm também sido apontados como potenciais causadores de dislipidémia. ${ }^{25}$

A insulinorresistência, a pré-diabetes e a diabetes mellitus tipo 2 representam um espectro de gravidade crescente das possíveis manifestações da disrupção do metabolismo glicídico nos doentes seropositivos medicados. Os inibidores da protease e os inibidores da transcriptase reversa análogos de nucleosídeos são os fármacos geralmente envolvidos nestas alterações. Os seus efeitos são mediados pela inibição do transportador transmembranar da glicose tipo 4 (GLUT4) presente nos miócitos e adipócitos; a redução da função do transportador dificulta a entrada de glicose mediada por insulina nestes tecidos conduzindo, numa fase inicial, a um aumento compensatório da síntese e secreção da insulina pancreática (hiperinsulinismo). Em idade pediátrica é raro encontrar-se diabetes mellitus tipo 2 , todavia as outras formas de alteração da homeostasia glicídica podem estar presentes, associando-se frequentemente a lipodistrofia e a síndrome metabólica. ${ }^{25,26,40-47}$

Os diferentes fatores de risco cardiovascular mencionados têm contudo falhado em explicar toda a tendência aterogénica presente nestes doentes e evidência científica corrobora que a predisposição aterosclerótica se mantém, mesmo após a correção dos fatores de risco convencionais. ${ }^{48,49}$

O estudo SMART é exemplo do exposto..$^{50,51}$ Os investigadores do estudo tentaram reduzir a toxicidade da terapêutica antirretrovírica e dos seus efeitos cardiovasculares, através do uso limitado destes fármacos, os quais seriam administrados apenas a doentes com contagem de células CD4 inferiores a 250 células $/ \mu \mathrm{L}$. A ideia subjacente era que uma menor exposição farmacológica, conduziria a menor lipodistrofia, dislipidémia e insulinorresistência e portanto a menor risco cardiovascular. Verificou-se porém o oposto, com um maior número de eventos cardiovasculares no braço do estudo sujeito a terapêutica mais conservadora. Foi por isso necessário repensar todo o processo aterosclerótico no contexto da infeção VIH e encontrar vias aterogénicas alternativas, ligadas mais ao processo infecioso do que aos efeitos secundários da terapêutica. ${ }^{52}$

Seguindo esta linha de pensamento, comprovou-se a existência de uma estreita relação entre risco cardiovascular e o controlo da infeção $\mathrm{VIH}$, traduzido na virémia e contagem de células CD4 do indivíduo (Fig.1). Um baixo nível de células CD4 e um elevado número de cópias de RNA viral, representam um mau controlo da infeção e são parâmetros de risco independentes para evento cardiovascular futuro, nomeadamente enfarte do miocárdio. ${ }^{52-57}$

$\mathrm{O} \mathrm{VIH}$ per si parece pois ter um efeito pró-aterosclerótico mediado por lesão endotelial e disfunção vascular. Esta lesão parece resultar quer da ação citopática direta do vírus na célula endotelial, quer de um efeito indireto do mesmo, ao promover um estado inflamatório crónico e pró-coagulante. A replicação viral contínua e a consequente ativação imunológica propiciam a inflamação, que pode ainda ser potenciada pela reativação de vírus latentes, como o citomegalovírus. Isto traduz-se num aumento dos mediadores bioquímicos de inflamação, como a proteína $C$ reativa ultrassensível, as citocinas monocíticas (a interleucina-6 e o fator de necrose tumoral- $\alpha$ ), a mieloperoxidase e certas moléculas de adesão celular (ex. seletina-P).,58-61 Estudos em idade pediátrica confirmam a existência, nesta faixa etária, de um ambiente pró-inflamatório, que se correlaciona com o aumento da espessura íntima-média carotídea. ${ }^{5,62}$

A presença de um estado pró-coagulante foi também identificada em crianças e adolescentes infetados. A replicação viral associa-se a uma deficiência de fatores anticoagulantes, como as proteínas S e C, permitindo criar um ambiente trombofílico, com aumento dos níveis séricos de D-dímeros. ${ }^{63}$

É desta amálgama interatuante de fatores que resulta a aterosclerose acelerada nos doentes seropositivos. A prevenção cardiovascular destes doentes deve, por isso, privilegiar uma atuação concertada de forma a reduzir o potencial nefasto dos diferentes protagonistas e pôr cobro à evolução aterosclerótica (Fig. 2).

\section{Marcadores vasculares de aterosclerose sub-clínica}

$\mathrm{Na}$ atualidade, a progressão da ateroslerose, nos doentes seropositivos e em outros grupos de risco, pode ser monitorizada de forma não invasiva, desde fases muito precoces e antes do aparecimento de qualquer sintoma associado. Recorre-se para isso a diferentes técnicas que permitem informar, quer sobre a estrutura e propriedades mecânicas da parede arterial, quer sobre a função endotelial. De facto, a aterogénese é acompanhada por um espessamento da parede arterial, por uma diminuição da elasticidade vascular e por uma perda de certas propriedades endoteliais. Estas alterações são passíveis de ser identificadas e quantificadas atribuindo-se, em idade pediátrica, um valor de percentil (ou z-score) respeitante à distribuição da variável em causa, tendo em conta o sexo, a idade e/ou estatura. ${ }^{64}$ 


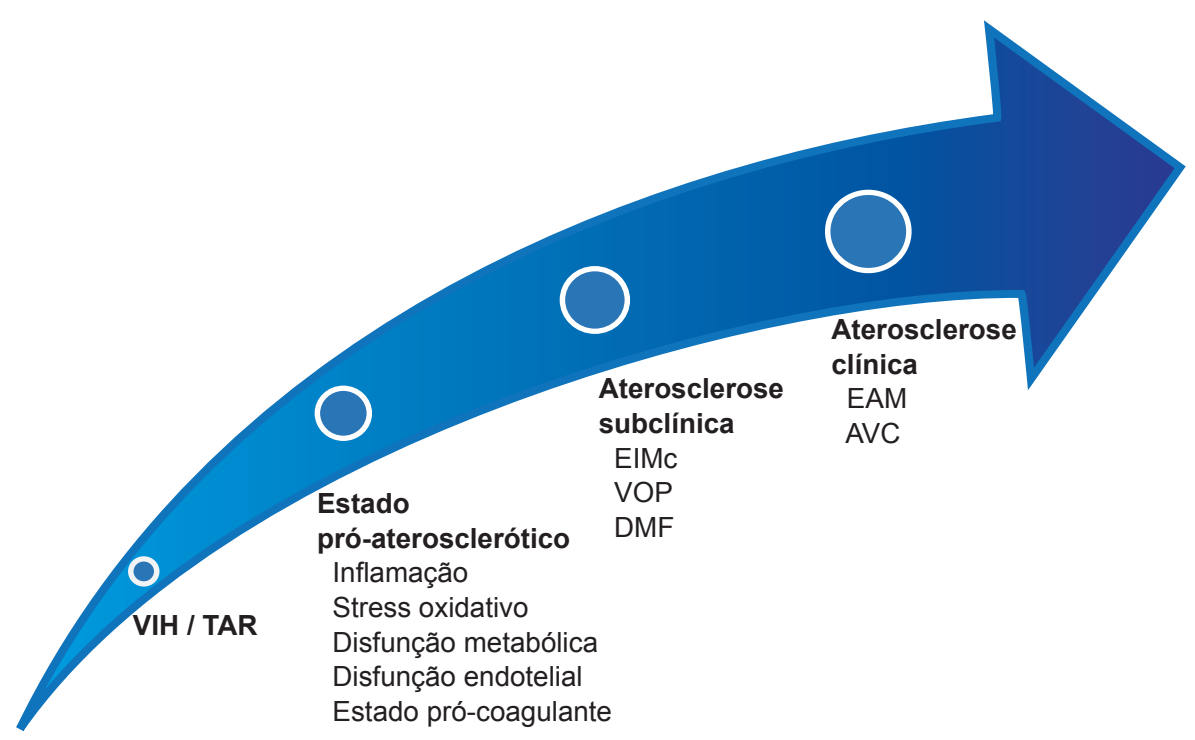

Figura 2 - Evolução da aterosclerose na infeção pelo vírus da imunodeficiência humana (VIH). A própria infeção e a terapêutica antirretrovírica (TAR) propiciam a existência de um ambiente pró-aterosclerótico, mediado por inflamação, stress oxidativo, disfunção metabólica, disfunção endotelial e estado pró-coagulante. Precocemente surgem lesões arteriais sub-clínicas, identificáveis por um aumento da espessura íntima-média carotídea, por um aumento da velocidade da onda-pulso e/ou compromisso da resposta vasodilatadora mediada pelo fluxo. Estas lesões iniciais correlacionam-se com a incidência de eventos cardiovasculares na idade adulta.

EIMc: espessura íntima-média carotídea; VOP: velocidade da onda de pulso; DMF: dilatação mediada por fluxo; EAM: enfarte agudo do miocárdio; AVC: acidente vascular cerebral

Um dos métodos mais comummente usados é a medição ecográfica da espessura da íntima-média carotídea (EIMc). Para essa determinação, usa-se uma sonda linear de ultrassons, colocada na região cervical e orientada sobre o trajeto longitudinal da artéria carótida comum. Consegue-se assim obter uma imagem de duas linhas ecogénicas que delimitam o espaço correspondente à camada íntima e camada média da parede arterial, passível de ser mensurado. ${ }^{65}$ Trata-se de um teste rápido, económico e reprodutível que auxilia na estratificação do risco cardiovascular, uma vez que a EIMc se correlaciona com eventos cardiovasculares futuros. ${ }^{66} \mathrm{Em}$ diferentes doenças pediátricas associadas a aterogénese precoce e acelerada, este marcador vascular tem-se demonstrado significativamente aumentado e capaz de traduzir a eficácia das terapêuticas anti-ateroscleróticas instituídas sobre o leito vascular. ${ }^{67}$

Remetendo-nos para a população pediátrica seropositiva, a EIMc encontra-se também aumentada; porém, uma intervenção privilegiando o controlo metabólico, a supressão viral e a restauração imunológica do indivíduo ajudam a suprimir esta tendência pró-aterosclerótica, aproximando o risco cardiovascular destes doentes ao da população em geral. $^{5-9}$

A par com a EIMc, outros meios auxiliares de diagnóstico podem ser utilizados para complementar e corroborar a informação obtida sobre o estado aterosclerótico. De particular interesse é a avaliação da rigidez arterial, que é aferida com recurso a diversas técnicas.

Uma das mais amplamente difundidas é a velocidade da onda de pulso (VOP), que se baseia no facto de a aterosclerose aumentar a rigidez arterial, o que por sua vez aumenta a rapidez com que a onda de pulso se propaga entre uma artéria proximal (ex. artéria carótida comum) e uma outra de localização mais distal (ex. femoral). Existem instrumentos que permitem captar a onda de pulso nestas duas artérias e a partir daí calcular o tempo que o sangue demora a percorrer a distância entre elas.$^{68} \mathrm{~A}$ velocidade de onda-pulso está aumentada nas crianças e adolescentes infetados e esta alteração parece tornar-se mais evidente com o aumento da idade, sobretudo no sub-grupo sob tratamento anti-retroviral. ${ }^{69-70}$

Por outro lado, a função do endotélio e a sua capacidade de produzir óxido nítrico podem também estar comprometidas no processo aterosclerótico. $\mathrm{O}$ grau de disfunção endotelial é quantificado pela medição da resposta vasodilatadora da artéria braquial ao aumento do fluxo sanguíneo, após desinsuflação de uma braçadeira de esfigmomanómetro. Este fenómeno de dilatação mediada por fluxo (DMF) é dependente do endotélio e da disponibilidade do óxido nítrico. A hiperémia reativa assim obtida, resultante da ação vasodilatadora do óxido nítrico, é depois comparada com a resposta vasodilatadora induzida pela administração sub-lingual de nitroglicerina, a qual não é mediada pelo endotélio. As crianças seropositivas apresentam uma dilatação mediada pelo fluxo significativamente diminuída face a grupos controlo mantendo-se, contudo, íntegra a resposta vasodilatadora não mediada pelo endotélio, sugerindo um compromisso da função do endotélio vascular. ${ }^{4,70}$

Não sendo aplicável o score de Framingham em idade pediátrica, estes marcadores vasculares de aterosclerose sub-clínica afiguram-se, num futuro próximo, como apetecíveis ferramentas para estratificação do risco cardiovascular nesta faixa etária. A sua correta valorização no contexto clínico carece ainda de evidência mais robusta proveniente de estudos longitudinais, que permita correlacionar de forma precisa os diferentes marcadores com uma 
probabilidade específica de evento cardiovascular futuro.

\section{Abordagem clínica}

Em 2006, um painel de peritos da American Heart Association publicou na revista Circulation, uma série de recomendações relativas à redução do risco cardiovascular em crianças com propensão para um processo aterogénico acelerado $^{71}$. Nesse documento, focou-se a atenção em diversas patologias pediátricas associadas a doença cardiovascular prematura. Tendo como sustentação os dados da medicina baseada na evidência, estes peritos distribuíram as patologias selecionadas por três grupos que estratificam o risco de doença coronária precoce (Tabela 1).

Esta classificação tem ainda em conta a existência concomitante de outros fatores de risco, nomeadamente: dislipidémia, tabagismo, história familiar de doença coronária precoce em parente de primeiro grau (homem antes dos 55 anos; mulher antes dos 65 anos), hipertensão arterial, insulinorresistência, sedentarismo, excesso de peso ou obesidade.

A presença simultânea de dois ou mais destes fatores implica automaticamente que o risco cardiovascular da criança suba para o patamar imediatamente acima, sendo as orientações terapêuticas pautadas por este novo nível de risco.

Nestas linhas de orientação, a infeção VIH ainda não era contemplada como pertencente a um dos grupos de risco mencionados. No entanto, a evidência crescente da sua associação com aterogénese prematura, fez com que em 2011, ela fosse incluída no grupo II ou de risco moderado, ${ }^{72}$ com possibilidade de passar para o grupo I ou de alto risco, se a criança ou o adolescente tiver dois ou mais dos fatores de risco cardiovascular descritos.

O risco cardiovascular existente torna imperioso atuar precocemente nestas crianças, no sentido de controlar eficazmente a infeção VIH e promover estilos de vida saudável. Criar o gosto pela prática de exercício físico e por uma dieta equilibrada é fundamental para evitar a obesidade e as co-morbilidades da síndrome metabólica. Igualmente importante é facultar informação sobre os malefícios do tabagismo e propiciar as condições necessárias para a desabituação tabágica nos adolescentes fumadores.

Para além destas medidas preventivas genéricas, recomenda-se uma monitorização regular de certos fatores de

Tabela 1 - Estratificação das diferentes doenças pediátricas de acordo com o risco cardiovascular associado

\begin{tabular}{|c|c|c|c|}
\hline Grupo & Categoria de risco & Perfil do grupo & Doenças \\
\hline Grupo I & Elevado & $\begin{array}{l}\text { Evidência clínica de doença } \\
\text { coronária antes dos } 30 \text { anos }\end{array}$ & $\begin{array}{l}\text { Hipercolesterolémia familiar homozigótica } \\
\text { Diabetes mellitus tipo } 1 \\
\text { Doença renal crónica/doença renal terminal } \\
\text { Transplante cardíaco } \\
\text { Doença de Kawasaki com aneurismas coronários mantidos }\end{array}$ \\
\hline Grupo II & Moderado & $\begin{array}{l}\text { Evidência fisiopatológica de } \\
\text { aterosclerose acelerada }\end{array}$ & $\begin{array}{l}\text { Hipercolesterolémia familiar heterozigótica } \\
\text { Diabetes mellitus tipo } 2 \\
\text { Infeção VIH } \\
\text { Doença inflamatória crónica } \\
\text { Doença de Kawasaki com aneurismas coronários que regrediram }\end{array}$ \\
\hline Grupo III & Em risco & $\begin{array}{l}\text { Evidência epidemiológica } \\
\text { de situação de risco para } \\
\text { aterosclerose acelerada }\end{array}$ & $\begin{array}{l}\text { Pós-terapêutica de doença oncológica } \\
\text { Cardiopatias congénitas } \\
\text { Doença de Kawasaki sem envolvimento coronário }\end{array}$ \\
\hline
\end{tabular}

Adaptado de Expert Panel on Integrated Guidelines for Cardiovascular Health and Risk Reduction in Children and Adolescents (2011)

Tabela 2 - Esquema de monitorização de diferentes parâmetros que influenciam o risco cardiovascular

\section{Esquema de monitorização}

Índice de massa corporal Em cada consulta

Pressão arterial

Sinais de lipodistrofia

Controlo da infeção VIH

- Virémia

- Contagem de CD4

Ficha lipídica

Metabolismo glicídico
Em cada consulta

Avaliar pelo menos uma vez por ano

Os dois parâmetros são avaliados na $1^{\text {a }}$ consulta, antes de iniciar TAR e depois, em períodos regulares de 3 a 4 meses, ou sempre que se fizer alterações na terapêutica. Pode-se optar por um controlo mais espaçado (seis a 12 meses) nas crianças e jovens com boa adesão à terapêutica, com supressão viral mantida, com níveis de CD4 muito acima do risco de infeção oportunística e estado clínico estável há mais de dois ou três anos.

A ficha lipídica em jejum deve ser obtida na primeira consulta, antes de iniciar TAR e depois a cada seis a 12 meses. Se houver antecedentes de valores lipídicos alterados, pode ser necessário avaliações em intervalos mais curtos.

Glicémia em jejum deve ser avaliada na primeira consulta, antes de iniciar TAR, três a seis meses após o seu início e depois, anualmente ou sempre que se fizer alterações na terapêutica. Se glicémias $\geq$ $140 \mathrm{mg} / \mathrm{dL}$, fazer medição após oito horas de jejum. Considerar referenciação para endocrinologista se valores alterados. 
risco cardiovasculares de acordo com o esquematizado na Tabela $2 .^{73}$

A agressividade com que os fatores de risco devem ser abordados depende do patamar de risco atribuído à criança. Para o nível II, aconselha-se um índice de massa corporal inferior ao percentil 90, uma pressão arterial sistólica e diastólica inferior ao percentil 95 e um colesterol LDL inferior a $130 \mathrm{mg} / \mathrm{dL}$. Por sua vez, as crianças pertencentes ao nível I devem ter um índice de massa corporal inferior ao percentil 85, uma pressão arterial inferior ao percentil 90 e um colesterol LDL inferior a $100 \mathrm{mg} / \mathrm{dL}^{.71}$

A primeira abordagem no sentido de alcançar os limites propostos consiste, como já referido, na adoção de estilos de vida mais saudáveis. Se esta medida não for contudo suficiente, a terapêutica farmacológica deve ser ponderada e considerada caso a caso.

\section{CONCLUSÃO}

A infeção VIH continua, na atualidade, a ser uma doença incurável, mas tratável. Ao longo dos mais de trinta anos decorridos desde o início do surto epidémico foi possível assistir ao aumento da sobrevida da população seropositiva. Este avanço foi possibilitado pelos fármacos anti-retrovirais e fez mudar o foco da atenção médica para as complicações desenvolvidas a longo prazo, como as doenças cardiovasculares. Investir na sua prevenção, nomeadamente em idade pediátrica, deve ser uma das preocupações dos clínicos responsáveis pelo seguimento destas crianças. Espera-se, contudo, que uma melhor perceção do processo aterosclerótico neste contexto, permita elaborar linhas de orientação clínicas cada vez mais eficazes e assim, aproximar ainda mais a esperança de vida dos infetados da existente na população em geral.

\section{CONFLITOS DE INTERESSE}

Os autores declaram não terem qualquer conflito de interesse relativamente ao presente artigo.

\section{FONTES DE FINANCIAMENTO}

Os autores declaram não terem beneficiado de qualquer financiamento.

\section{REFERÊNCIAS}

1. Stramba-Badiale M, Fox KM, Priori SG, Collins P, Daly C, Graham I, et al. Cardiovascular diseases in women: a statement from the policy conference of the European Society of Cardiology. Eur Heart J. 2006;27:994-1005.

2. Freiberg MS, Chang CC, Kuller LH, Skanderson M, Lowy E, Kraemer $\mathrm{KL}$, et al. HIV infection and the risk of acute myocardial infarction. JAMA Intern Med. 2013;173:614-22.

3. Marcus JL, Leyden WA, Chao CR, Chow FC, Horberg MA, Hurley LB, et al. HIV infection and incidence of ischemic stroke. AIDS. 2014;28:19119.

4. Charakida M, Donald A, Green H, Storry C, Clapson M, Caslake M, et al. Early structural and functional changes of the vasculature in HIV-infected children - Impact of disease and antiretroviral therapy. Circulation. 2005;112:103-9.

5. McComsey GA, O'Riordan M, Hazen SL, El-Bejjani D, Bhatt S, Brennan $\mathrm{ML}$, et al. Increased carotid intima media thickness and cardiac biomarkers in HIV infected children. AIDS. 2007;21:921-7.

6. Giuliano IC, de Freitas SF, de Souza M, Caramelli B. Subclinic atherosclerosis and cardiovascular risk factors in HIV-infected children: PERI study. Coron Artery Dis. 2008;19:167-72.

7. Ross AC, Storer N, O'Riordan MA, Dogra V, McComsey GA. Longitudinal changes in carotid intima-media thickness and cardiovascular risk factors in human immunodeficiency virus-infected children and young adults compared with healthy controls. Pediatr Infect Dis J. 2010;29:6348.

8. Hanna DB, Guo M, Bůžková P, Miller TL, Post WS, Stein JH, et al. HIV infection and carotid artery intima-media thickness: pooled analyses across 5 cohorts of the NHLBI HIV-CVD Collaborative. Clin Infect Dis. 2016;63:249-56.

9. Chanthong P, Lapphra K, Saihongthong S, Sricharoenchai S, Wittawatmongkol O, Phongsamart W, et al. Echocardiography and carotid intima-media thickness among asymptomatic HIV-infected adolescents in Thailand. AIDS. 2014;28:2071-9.

10. Bonnet D, Aggoun Y, Szezepanski I, Bellal N, Blanche S. Arterial stiffness and endothelial dysfunction in HIV-infected children. AIDS. 2004;18:1037-41.

11. Gritz ER, Vidrine DJ, Lazev AB, Amick BC $3^{\text {rd }}$, Arduino RC. Smoking behavior in a low-income multiethnic HIVIAIDS population. Nicotine Tob Res. 2004;6:71-7.

12. Mamary EM, Bahrs D, Martinez S. Cigarette smoking and the desire to quit among individuals living with HIV. AIDS Patient Care STDS. 2002;16:39-42.

13. Friis-Moller $\mathrm{N}$, Weber $\mathrm{R}$, Reiss $\mathrm{P}$, Thiébaut $\mathrm{R}$, Kirk $\mathrm{O}$, d'Arminio

Monforte A, et al. Cardiovascular disease risk factors in HIV patientsassociation with antiretroviral therapy. Results from the DAD study. AIDS. 2003;17:1179-93.

14. Saves M, Chene G, Ducimetiere P, Leport C, Le Moal G, Amouyel P, et al. Risk factors for coronary heart disease in patients treated for human immunodeficiency virus infection compared with the general population. Clin Infect Dis. 2003;37:292-8.

15. Palacios R, Santos J, Garcia A, Castells E, González M, Ruiz J, et al. Impact of highly active antiretroviral therapy on blood pressure in HIVinfected patients. A prospective study in a cohort of naive patients. HIV Med. 2006;7:10-5.

16. Seaberg EC, Munoz A, Lu M, Detels R, Margolick JB, Riddler SA, et al. Association between highly active antiretroviral therapy and hypertension in a large cohort of men followed from 1984 to 2003. AIDS. 2005;19:953-60.

17. Dimala CA, Atashili J, Mbuagbaw JC, Wilfred A, Monekosso GL. Prevalence of hypertension in HIVIAIDS patients on highly active antiretroviral therapy (HAART) compared with HAART-naïve patients at the Limbe Regional Hospital, Cameroon. PLoS One. 2016;11:e0148100.

18. Gazzaruso C, Bruno R, Garzaniti A, Giordanetti S, Fratino P, Sacchi P, et al. Hypertension among HIV patients: prevalence and relationships to insulin resistance and metabolic syndrome. J Hypertension. 2003;21:1377-82.

19. Thiébaut R, El-Sadr WM, Friis-Møller $N$, Rickenbach $M$, Reiss $P$, Monforte AD, et al. Predictors of hypertension and changes of blood pressure in HIV-infected patients. Antivir Ther. 2005;10:811-23.

20. de Arruda Junior ER, Lacerda HR, Moura LC, Albuquerque MF, Miranda Filho DB, Diniz GT, et al. Risk factors related to hypertension among patients in a cohort living with HIVIAIDS. Braz J Infect Dis. 2010;14:2817.

21. Crane HM, Van Rompaey SE, Kitahata MM. Antiretroviral medications associated with elevated blood pressure among patients receiving highly active antiretroviral therapy. AIDS. 2006;20:1019-26.

22. Jericó $\mathrm{C}$, Knobel H, Montero M, Sorli ML, Guelar A, Gimeno JL, et al. Hypertension in HIV-infected patients: prevalence and related factors. Am J Hypertens. 2005;18:1396-401.

23. Bergersen BM, Sandvik L, Dunlop O, Birkeland K, Bruun JN. Prevalence of hypertension in HIV-positive patients on highly active retroviral therapy (HAART) compared with HAART-naive and HIV-negative controls: results from a Norwegian study of 721 patients. Eur J Clin Microbiol Infect Dis. 2003;22:731-6.

24. Chatterton-Kirchmeier S, Camacho-Gonzalez AF, McCracken CE, Chakraborty R, Batisky DL. Increased prevalence of elevated blood 
pressures in HIV-infected children, adolescents and young adults. Pediatr Infect Dis J. 2015;34:610-4

25. Panel on Antiretroviral Therapy and Medical Management of HIVInfected Children. Guidelines for the Use of Antiretroviral Agents in Pediatric HIV Infection. [consultado 2016 nov 1]. Disponível em https:// aidsinfo.nih.gov/contentfiles/lvguidelines/pediatricguidelines.pdf. (tabelas 12b, 12h).

26. Barlow-Mosha L, Eckard AR, McComsey GA, Musoke PM. Metabolic complications and treatment of perinatally HIV-infected children and adolescents. J Int AIDS Soc. 2013;16:18600.

27. Parakh A, Dubey AP, Kumar A, Maheshwari A. Lipodystrophy and metabolic complications of highly active antiretroviral therapy. Indian J Pediatr. 2009;76:1017-21.

28. Barbaro G. Metabolic and cardiovascular complications of highly active antiretroviral therapy for HIV infection. Curr HIV Res. 2006;4:79-85.

29. Hadigan C, Meigs JB, Corcoran C, Rietschel P, Piecuch S, Basgoz N, et al. Metabolic abnormalities and cardiovascular disease risk factors in adults with human immunodeficiency virus infection and lipodystrophy. Clin Infect Dis. 2001;32:130-9.

30. Werner ML, Pone MV, Fonseca VM, Chaves CR. Lipodystrophy syndrome and cardiovascular risk factors in children and adolescents infected with HIVIAIDS receiving highly active antiretroviral therapy. J Pediatr. 2010;86:27-32

31. Papia L, Menezes AC, Rocha H, Abreu T, Oliveira RH, Frota AC, et al. Prevalence of lipodystrophy and risk factors for dyslipidemia in HIVinfected children in Brazil. Braz J Infect Dis. 2014;18:394-9.

32. Innes S, Cotton MF, Haubrich R, Conradie MM, Van Niekerk M, Edson C, et al. High prevalence of lipoatrophy in pre-pubertal South African children on antiretroviral therapy: a cross-sectional study. BMC Pediatr. 2012;12:183.

33. Piloya T, Bakeera-Kitaka S, Kekitiinwa A, Kamya MR. Lipodystrophy among HIV-infected children and adolescents on highly active antiretroviral therapy in Uganda: a cross sectional study. J Int AIDS Soc. 2012;15:17427

34. Bwakura-Dangarembizi $M$, Musiime V, Szubert AJ, Prendergast AJ, Gomo ZA, Thomason MJ, et al. Prevalence of lipodystrophy and metabolic abnormalities in HIV-infected African children after 3 years on first-line antiretroviral therapy. Pediatr Infect Dis J. 2015;34:e23-31.

35. Prendergast AJ. Complications of long-term antiretroviral therapy in HIVinfected children. Arch Dis Child. 2013;98:245-6.

36. Farley J, Gona P, Crain M, Cervia J, Oleske J, Seage G, et al. Prevalence of elevated cholesterol and associated risk factors among perinatally HIV-infected children (4-19 years old) in Pediatric AIDS Clinical Trials Group 219C. J Acquir Immune Defic Syndr. 2005;38:480-7.

37. Tassiopoulos K, Williams PL, Seage GR III, Crain M, Oleske J, Farley $\mathrm{J}$, et al. Association of hypercholesterolemia incidence with antiretroviral treatment, including protease inhibitors, among perinatally HIV-infected children. J Acquir Immune Defic Syndr. 2008;47:607-14

38. Rhoads MP, Lanigan J, Smith CJ, Lyall H. Effect of specific antiretroviral therapy (ART) drugs on lipid changes and the need for lipid management in children with HIV. J Acquir Immune Defic Syndr. 2011;57:404-12.

39. Tran H, Robinson S, Mikhailenko I, Strickland DK. Modulation of the LDL receptor and LRP levels by HIV protease inhibitors. J Lipid Res. 2003;44:1859-69.

40. Brown TT, Cole SR, Li X, Kingsley LA, Palella FJ, Riddler SA, et al. Antiretroviral therapy and the prevalence and incidence of diabetes mellitus in the multicenter AIDS cohort study. Arch Intern Med. 2005; 165:1179-84.

41. Alvaro-Meca A, Jiménez-Garcia R, Jimenez-Trujillo I, HernandezBarrera V, de Miguel-Diez J, Resino S, et al. Fifteen-year trends in the prevalence of diabetes among hospitalized HIV-infected patients in Spain (1997-2012). PLoS One. 2016;11:e0161953.

42. Gutierrez AD, Balasubramanyam A. Dysregulation of glucose metabolism in HIV patients: epidemiology, mechanisms, and management. Endocrine. 2012;41:1-10.

43. Viganò A, Brambilla P, Pattarino G, Stucchi S, Fasan S, Raimondi C, et al. Long-term evaluation of glucose homeostasis in a cohort of HAARTtreated HIV-infected children: a longitudinal, observational cohort study. Clin Drug Investig. 2009;29:101-9.

44. Lee B, Aurpibul L, Sirisanthana V, Mangklabruks A, Sirisanthana T, Puthanakit T. Low prevalence of insulin resistance among HIV-infected children receiving nonnucleoside reverse transcriptase inhibitor-based highly active antiretroviral therapy in Thailand. HIV Med. 2009;10:72-8.

45. Hazra R, Hance LF, Monteiro JP, Ruz NP, Machado DM, Saavedra M, et al. Insulin resistance and glucose and lipid concentrations in a cohort of perinatally HIV-infected Latin American children. Pediatr Infect Dis J.
2013:32:757-9.

46. Blázquez D, Ramos-Amador JT, Saínz T, Mellado MJ, García-Ascaso $\mathrm{M}$, De José MI, et al. Lipid and glucose alterations in perinatallyacquired HIV-infected adolescents and young adults. BMC Infect Dis. 2015;15:119.

47. dos Reis LC, de Carvalho Rondó $\mathrm{PH}$, de Sousa Marques $\mathrm{HH}$, de Andrade SB. Dyslipidaemia and insulin resistance in vertically HIV-infected children and adolescents. Trans R Soc Trop Med Hyg. 2011;105:197203.

48. Triant VA. HIV infection and coronary heart disease: an intersection of epidemics. J Infect Dis. 2012;205:S355-61.

49. Hemkens LG, Bucher HC. HIV infection and cardiovascular disease. Eu Heart J. 2014;35:1373-81.

50. Strategies for Management of Antiretroviral Therapy (SMART) Study Group, El-Sadr WM, Lundgren J, Neaton JD, Gordin F, Abrams D, et al. CD4+ count-guided interruption of antiretroviral treatment. $\mathrm{N}$ Engl J Med. 2006;355:2283-96.

51. Strategies for Management of Antiretroviral Therapy (SMART) Study Group, Lundgren JD, Babiker A, El-Sadr W, Emery S, Grund B, et al. Inferior clinical outcome of the CD4+ cell count-guided antiretroviral treatment interruption strategy in the SMART study: role of CD4+ Cell counts and HIV RNA levels during follow-up. J Infect Dis. 2008;197:114555.

52. Freiberg MS, Chang CC, Kuller LH, Skanderson M, Lowy E, Kraemer $\mathrm{KL}$, et al. HIV infection and the risk of acute myocardial infarction. JAMA Intern Med. 2013;173:614-22

53. Silverberg MJ, Leyden WA, Xu L, Horberg MA, Chao CR, Towner WJ, et al. Immunodeficiency and risk of myocardial infarction among HIVpositive individuals with access to care. J Acquir Immune Defic Syndr. 2014;65:160-6.

54. Lang S, Mary-Krause M, Simon A, Partisani M, Gilquin J, Cotte L, et al HIV replication and immune status are independent predictors of the risk of myocardial infarction in HIV-infected individuals. Clin Infect Dis. 2012; 55:600-7.

55. Lichtenstein KA, Armon C, Buchacz K, Chmiel JS, Buckner K, Tedald $\mathrm{EM}$, et al. Low CD4+ $\mathrm{T}$ cell count is a risk factor for cardiovascular disease events in the HIV outpatient study. Clin Infect Dis. 2010;51:43547.

56. Triant VA, Regan S, Lee H, Sax PE, Meigs JB, Grinspoon SK. Association of immunologic and virologic factors with myocardial infarction rates in a U.S. Health Care system. J Acquir Immune Defic Syndr. 2010;55:615-9.

57. Blum A, Hadas V, Burke M, Yust I, Kessler A. Viral load of the human immunodeficiency virus could be an independent risk factor for endothelial dysfunction. Clin Cardiol. 2005;28:149-53.

58. De Luca A, de Gaetano Donati K, Colafigli M, Cozzi-Lepri A, De Curtis A, Gori A, et al. The association of high-sensitivity c-reactive protein and other biomarkers with cardiovascular disease in patients treated for HIV: a nested case-control study. BMC Infect Dis. 2013;13:414

59. Masiá M, Bernal E, Padilla S, Graells ML, Jarrín I, Almenar MV, et al. The role of $\mathrm{C}$-reactive protein as a marker for cardiovascular risk associated with antiretroviral therapy in HIV-infected patients. Atherosclerosis. 2007; 195:167-71.

60. Miller TL, Somarriba G, Orav EJ, Mendez AJ, Neri D, Schaefer N, et al. Biomarkers of vascular dysfunction in children infected with human immunodeficiency virus-1. J Acquir Immune Defic Syndr. 2010;55:182-

61. Miller TI, Borkowsky W, DiMeglio LA, Dooley L, Geffner ME, Hazra R, et al. Metabolic abnormalities and viral replication are associated with biomarkers of vascular dysfunction in HIV-infected children. HIV Med. 2012;13:264-75.

62. Ross AC, O'Riordan MA, Storer N, Dogra V, McComsey GA. Heightened inflammation is linked to carotid intima-media thickness and endothelial activation in HIV-infected children. Atherosclerosis. 2010;211:492-8.

63. Pontrelli G, Martino AM, Tchidjou HK, Citton R, Mora N, Ravà L, et al. $\mathrm{HIV}$ is associated with thrombophilia and high D-dimer in children and adolescents. AIDS. 2010; 24:1145-51.

64. Doyon A, Kracht D, Bayazit AK, Deveci M, Duzova A, Krmar RT, et al. Carotid artery intima-media thickness and distensibility in children and adolescents: reference values and role of body dimensions. Hypertension. 2013; 62:550-6.

65. Dalla Pozza R, Ehringer-Schetitska D, Fritsch P, Jokinen E, Petropoulos A, Oberhoffer R, et al. Intima media thickness measurement in children: a statement from the Association for European Paediatric Cardiology (AEPC) Working Group on Cardiovascular Prevention endorsed by the Association for European Paediatric Cardiology. Atherosclerosis. $2015 \cdot 238: 380-7$ 
66. Robertson CM, Gerry F, Fowkes R, Price JF. Carotid intima-media thickness and the prediction of vascular events. Vasc Med. 2012;17:23948.

67. Litwin M, Niemirska A. Intima-media thickness measurements in children with cardiovascular risk factors. Pediatr Nephrol. 2009;24:707-19.

68. Aggoun Y, Szezepanski I, Bonnet D. Noninvasive assessment of arterial stiffness and risk of atherosclerotic events in children. Pediatr Res. 2005;58:173-8.

69. Charakida M, Loukogeorgakis SP, Okorie MI, Masi S, Halcox JP, Deanfield JE, et al. Increased arterial stiffness in HIV-infected children: risk factors and antiretroviral therapy. Antivir Ther. 2009;14:1075-9.

70. Bonnet D, Aggoun Y, Szezepanski I, Bellal N, Blanche S. Arterial stiffness and endothelial dysfunction in HIV-infected children. AIDS. 2004;18:1037-41.

71. Kavey RE, Allada V, Daniels SR, Hayman LL, McCrindle BW, Newburger $\mathrm{JW}$, et al. Cardiovascular risk reduction in high-risk pediatric patients: a scientific statement from the American Heart Association Expert Panel on Population and Prevention Science; the Councils on Cardiovascular Disease in the Young, Epidemiology and Prevention, Nutrition, Physical Activity and Metabolism, High Blood Pressure Research, Cardiovascular Nursing, and the Kidney in Heart Disease; and the Interdisciplinary Working Group on Quality of Care and Outcomes Research. Circulation. 2006;114:2710-38.

72. Expert Panel on Integrated Guidelines for Cardiovascular Health and Risk Reduction in Children and Adolescents, National Heart, Lung, and Blood Institute. Expert Panel on Integrated Guidelines for Cardiovascular Health and Risk Reduction in Children and Adolescents: summary report. Pediatrics. 2011;128:S213-56.

73. Panel on Antiretroviral Therapy and Medical Management of HIVInfected Children. Guidelines for the Use of Antiretroviral Agents in Pediatric HIV Infection. [consultado 2016 dez 1]. Disponível em http:// aidsinfo.nih.gov/contentfiles/lvguidelines/pediatricguidelines.pdf. 